\title{
A note on convergence of semigroups
}

\author{
by ADAm BoBrowski (Lublin)
}

\begin{abstract}
Convergence of semigroups which do not converge in the Trotter-KatoNeveu sense is considered.

1. Introduction. The classical Trotter-Kato-Neveu theorem ([23], pp. 44-46, [34], p. 502 , [55], p. 269 , [48], p. $87,[20]$, p. 83, [46]) states that a sequence $\left\{T_{n}(t): t \geq 0\right\}, n \geq 1$, of equibounded semigroups $\left(\left\|T_{n}(t)\right\| \leq M\right)$ converges strongly and almost uniformly with respect to $t \in \mathbb{R}^{+}:=[0, \infty)$ iff their resolvents $R_{\lambda, n}$ converge strongly and the image Range $R_{\lambda}$ of the limit pseudoresolvent is dense in the Banach space $L$ they act in.

If $L^{\prime}:=\overline{\text { Range } R_{\lambda}} \neq L$, the assertion of the theorem remains valid for $f \in L^{\prime}$ ([38], [16], [23], p. 34, or [10-11]), but in general for $f \notin L^{\prime}$ the limit $\lim _{n \rightarrow \infty} T_{n}(t) f$ may exist neither in the strong nor in the weak topology ([9], p. 319). This is due to the fact that the convergence of the resolvents of the semigroups $\left\{T_{n}(t): t \geq 0\right\}$ alone is equivalent to the convergence of Lipschitz continuous once-integrated semigroups $\int_{0}^{t} T_{n}(s) d s$, rather than the semigroups themselves ([38], p. 29, Prop. $(2-22)$ or $[11,15])$, or as noted by J. Kisyński (personal communication), to the convergence of all sequences of the form $\int_{0}^{\infty} \phi(t) T_{n}(t) f d t$ where $\phi \in \mathcal{L}^{1}\left(\mathbb{R}^{+}\right)$and $f \in L$ (cf. also [8], Th. 4.1, and Th. 3 below).

On the other hand, a considerable number of instances of semigroups have been found converging for all $f \in L$ in spite of the fact that $L^{\prime} \neq L$ $([9-10])$. No source of this phenomenon, general enough to cover all important examples, seems to be known; in [9-10] different arguments were used for each case separately. The similarity of this phenomenon to the loss of regularity during the process of convergence, as studied e.g. in $[1-3,14,27$, $53]$, is merely superficial, for the phenomenon in question is not related to weaker stability conditions, the semigroups remaining equibounded, but to
\end{abstract}

1991 Mathematics Subject Classification: 47D03, 41A35.

Key words and phrases: semigroup, approximation, differentiable semigroup, resolvent, asymptotic behaviour, mean ergodic theorems. 
the sole fact that $L^{\prime} \neq L$. The limit semigroup fails to belong to either of the known classes of semigroups, as presented in [32], since the domain of its infinitesimal generator is not dense in $L$ (however, compare [9] with [12, 19, 41-43]). Our knowledge of general properties of such a convergence is also rather limited.

This paper aims at answering some related questions. First of all, we prove that if the convergence holds for all $f \notin L^{\prime}$, then it is almost uniform in $(0, \infty)$ (Proposition 2). Furthermore, we provide a necessary and sufficient condition for the existence of such a limit in terms of Abel summability (Proposition 3), and a natural sufficient condition for semigroups in question to be equidifferentiable (Proposition 4), which, thanks to Pazy's characterization of generators of differentiable semigroups, can be expressed in terms of the resolvents of $\left\{T_{n}(t): t \geq 0\right\}$. Since the convergence of the resolvents of semigroups is necessary for the convergence of the semigroups, this gives a complete characterization of the convergence of equidifferentiable semigroups.

Section 3 is devoted to applications to the convergence of ergodic averages and asymptotic stability of bounded semigroups. It is shown that, in contrast to convergence in the Trotter-Kato-Neveu sense, theorems concerning the almost uniform (in $t \in(0, \infty)$ ) convergence of semigroups can be applied to prove results on the asymptotic behaviour of the semigroups, such as asymptotic stability or asymptotic decay.

For instance, it is proved that the existence of the $\operatorname{limit}_{t \rightarrow \infty} T(t) f$ for a holomorphic semigroup $\{T(t): t \geq 0\}$ is equivalent to the existence of $\lim _{\varepsilon \rightarrow 0} \varepsilon(\varepsilon-A)^{-1} f$ where $A$ is the generator of the semigroup.

Throughout the paper it is assumed that approximating semigroups and the limit semigroup act in the same Banach space. Although such a setting is not customary in approximation theory (see e.g. [48], p. 94), it fits well the applications presented in Section 3. It was chosen for simplicity with the hope that the reader will himself look for straightforward generalizations when needed.

2. The main results. Suppose we are given a sequence of equibounded semigroups $\left\{T_{n}(t): t \geq 0\right\},\left\|T_{n}(t)\right\| \leq M$, with respective generators $A_{n}$, acting in a complex Banach space $L$. Assume furthermore that

$$
\lim _{n \rightarrow \infty}\left(\lambda-A_{n}\right)^{-1} f=: R_{\lambda} f \quad \text { exists for all } f \in L \text { and } \operatorname{Re} \lambda>0 .
$$

This is equivalent to the apparently weaker assumption that the above limit exists for a fixed $\lambda$ with $\operatorname{Re} \lambda>0$ (see e.g. [48], p. 87, Th. 4.3).

We are interested in convergence of $\left\{T_{n}(t): t \geq 0\right\}$ (not necessarily almost uniform in $\mathbb{R}^{+}$). 
Let us begin by noting that, in contrast to the case of the Trotter-KatoNeveu theorem, the existence of the limit in question is not a property of the limit pseudoresolvent. This is proved by the following simple example.

EXAmple 1 (cf. [9], p. 319). Let $\{T(t): t \geq 0\}$ be a $C_{0}$ contraction semigroup acting in a Banach space $L^{\prime}$, and let

$$
L:=L^{\prime} \times \mathbb{C}, \quad\left\|\left(\begin{array}{l}
f \\
z
\end{array}\right)\right\|_{L}=\|f\|_{L^{\prime}}+|z| .
$$

The semigroups

$$
T_{n}(t)\left(\begin{array}{l}
f \\
z
\end{array}\right)=\left(\begin{array}{c}
T(t) f \\
e^{-n t z}
\end{array}\right) \quad \text { and } \quad S_{n}(t)\left(\begin{array}{l}
f \\
z
\end{array}\right)=\left(\begin{array}{c}
T(t) f \\
e^{i n t} z
\end{array}\right)
$$

acting in $L$ have the resolvents

$$
R_{\lambda, n}\left(\begin{array}{l}
f \\
z
\end{array}\right)=\left(\begin{array}{c}
(\lambda-A)^{-1} f \\
\frac{1}{\lambda+n} z
\end{array}\right) \quad \text { and } \quad R_{\lambda, n}^{\prime}\left(\begin{array}{l}
f \\
z
\end{array}\right)=\left(\begin{array}{c}
(\lambda-A)^{-1} f \\
\frac{1}{\lambda-i n} z
\end{array}\right)
$$

respectively, and the same limit pseudoresolvent

$$
R_{\lambda}\left(\begin{array}{l}
f \\
z
\end{array}\right)=\left(\begin{array}{c}
(\lambda-A)^{-1} f \\
0
\end{array}\right)
$$

where $A$ is the generator of $\{T(t): t \geq 0\}$. On the other hand, however,

$$
\lim _{n \rightarrow \infty} T_{n}(t)\left(\begin{array}{l}
f \\
z
\end{array}\right)=\left(\begin{array}{c}
T(t) f \\
0
\end{array}\right), \quad z \in \mathbb{C}, f \in L, t>0,
$$

while the corresponding limit of $\left\{S_{n}(t): t \geq 0\right\}$ exists only on $L^{\prime} \times\{0\}$.

Thus, by taking the limit of the resolvent we loose information, at least partially. The operator $\mathcal{A}$ in the Banach space $\mathcal{L}$ of all convergent sequences $\left(f_{n}\right)_{n \geq 1}, f_{n} \in L$, defined by $[35,38]$

$$
\begin{aligned}
& \mathcal{D}(\mathcal{A})=\left\{\left(f_{n}\right)_{n \geq 1} \in \mathcal{L}: f_{n} \in \mathcal{D}\left(A_{n}\right),\left(A_{n} f_{n}\right)_{n \geq 1} \in \mathcal{L}\right\}, \\
& \mathcal{A}\left(f_{n}\right)_{n \geq 1}=\left(A_{n} f_{n}\right)_{n \geq 1},
\end{aligned}
$$

provides us with much more data. The space $\mathcal{L}$ is equipped with the usual supremum norm $\left\|\left(f_{n}\right)_{n \geq 1}\right\|_{\mathcal{L}}=\sup _{n \in \mathbb{N}}\left\|f_{n}\right\|_{L}$.

By assumption (2.1), one can define a pseudoresolvent $\mathcal{R}_{\lambda}$ in $\mathcal{L}$ by

$$
\mathcal{R}_{\lambda}\left(f_{n}\right)_{n \geq 1}=\left(R_{\lambda, n} f_{n}\right)_{n \geq 1} \quad \text { for } \operatorname{Re} \lambda>0 .
$$

It can be checked that

$$
\begin{array}{ll}
\mathcal{R}_{\lambda}(\lambda-\mathcal{A})\left(f_{n}\right)_{n \geq 1}=\left(f_{n}\right)_{n \geq 1} & \text { for all }\left(f_{n}\right)_{n \geq 1} \in \mathcal{D}(\mathcal{A}), \\
(\lambda-\mathcal{A}) \mathcal{R}_{\lambda}\left(f_{n}\right)_{n \geq 1}=\left(f_{n}\right)_{n \geq 1} & \text { for all }\left(f_{n}\right)_{n \geq 1} \in \mathcal{L} .
\end{array}
$$

Thus, by equicontinuity of $\left\{T_{n}(t): t \geq 0\right\}$ we have

$$
\left\|(\lambda-\mathcal{A})^{-n}\right\| \leq M /(\operatorname{Re} \lambda)^{n} \quad \text { for } \operatorname{Re} \lambda>0 .
$$


The operator $\mathcal{A}$ is therefore closed, and by the Arendt theorem [4] (see also $[11,13,41-43]$ for simple alternative proofs), it is the infinitesimal generator of a Lipschitz continuous integrated semigroup $\{\mathcal{U}(t): t \geq 0\}$. One can prove that

$$
\mathcal{U}(t)\left(f_{n}\right)_{n \geq 1}=\left(\int_{0}^{t} T_{n}(s) f_{n} d s\right)_{n \geq 1} \quad \text { for all }\left(f_{n}\right)_{n \geq 1} \in \mathcal{L} .
$$

Furthermore, the operator $\mathcal{A}_{0}$, the part of $\mathcal{A}$ in $\mathcal{L}^{\prime}=\overline{\mathcal{D}(\mathcal{A})}$ (that is, $\mathcal{D}\left(\mathcal{A}_{0}\right)=$ $\left.\left\{\left(f_{n}\right)_{n \geq 1} \in \mathcal{D}(\mathcal{A}): \mathcal{A}\left(f_{n}\right)_{n \geq 1} \in \mathcal{L}^{\prime}\right\}, \mathcal{A}_{0}=\mathcal{A}_{\mid \mathcal{D}\left(\mathcal{A}_{0}\right)}\right)$ is the infinitesimal generator of a strongly continuous semigroup $\left\{\mathcal{T}_{0}(t): t \geq 0\right\}$ acting in $\mathcal{L}^{\prime}$ :

$$
\begin{gathered}
\mathcal{L}^{\prime}=\overline{\mathcal{D}(\mathcal{A})}=\overline{\text { Range }(\lambda-\mathcal{A})^{-1}}=\left\{\left(f_{n}\right)_{n \geq 1}: \lim _{n \rightarrow \infty} f_{n} \in \overline{\text { Range } R_{\lambda}}\right\}, \\
\mathcal{T}_{0}(t)\left(f_{n}\right)_{n \geq 1}=\left(T_{n}(t) f_{n}\right)_{n \geq 1} \quad \text { for }\left(f_{n}\right)_{n \geq 1} \in \mathcal{L}^{\prime} .
\end{gathered}
$$

Observe that the ranges above do not depend on $\lambda$ with $\operatorname{Re} \lambda>0$. The straightforward proof of these facts may be found in [11].

The following theorem was proved in [12].

Theorem 1. Let $\mathcal{A}$ be a closed operator in a Banach space $\mathcal{L}$ such that (2.3) is satisfied. Let $\mathcal{L}^{\prime} \subset \mathcal{L}$ be a subspace and $\left\{\mathcal{T}_{0}(t): t \geq 0\right\}$ be the strongly continuous semigroup acting in $\mathcal{L}^{\prime}$ generated by $\mathcal{A}_{0}$, the part of $\mathcal{A}$ in $\mathcal{L}^{\prime}$. The following statements are equivalent:

(i) there exists a unique strongly measurable semigroup $\{\mathcal{T}(t): t \geq 0\}$ (discontinuous at $t=0$ iff $\mathcal{L}^{\prime} \neq \mathcal{L}$ ) such that

$$
\mathcal{R}_{\lambda} \widetilde{f}=(\lambda-\mathcal{A})^{-1} \widetilde{f}=\int_{0}^{\infty} e^{-\lambda t} \mathcal{T}(t) \widetilde{f} d t \quad \text { for } \tilde{f} \in \mathcal{L}, \lambda>0,
$$

(ii) for all $\tilde{f}$ in a dense subset of $\mathcal{L}$ and $t>0$ the limit

$$
\lim _{\lambda \rightarrow \infty} \lambda \mathcal{T}_{0}(t) \mathcal{R}_{\lambda} \tilde{f} \quad(\lambda \text { real })
$$

exists,

(iii) the above limit exists for all $\tilde{f} \in \mathcal{L}$,

(iv) for all $\tilde{f} \in \mathcal{L}, \lambda>0$, and $t>0$,

$$
\mathcal{T}_{0}(t) \mathcal{R}_{\lambda} \tilde{f} \in \mathcal{D}\left(\mathcal{A}_{0}\right) .
$$

Furthermore, the semigroup $\{\mathcal{T}(t): t \geq 0\}$ in (i) is given by

$$
\mathcal{T}(t) \tilde{f}=\lim _{\lambda \rightarrow \infty} \lambda \mathcal{T}_{0}(t) \mathcal{R}_{\lambda} \tilde{f} \quad(\lambda \text { real }) .
$$

Proposition 1. The semigroups $\left\{T_{n}(t): t \geq 0\right\}$ converge for all $f \in L$ iff for the operator $\mathcal{A}$ defined by (2.2) there exists a semigroup $\{\mathcal{T}(t): t \geq 0\}$, acting in $\mathcal{L}$, described in Theorem 1. 
Proof. (The "if" part) By Theorem 1(i) we have $\left(\widetilde{f}=\left(f_{n}\right)_{n \geq 1}\right)$

$$
\begin{aligned}
\int_{0}^{\infty} e^{-\lambda t} \mathcal{T}(t)\left(f_{n}\right)_{n \geq 1} d t & =(\lambda-\mathcal{A})^{-1}\left(f_{n}\right)_{n \geq 1}=\left(R_{\lambda, n} f_{n}\right)_{n \geq 1} \\
& =\left(\int_{0}^{\infty} e^{-\lambda t} T_{n}(t) f_{n} d t\right)_{n \geq 1}
\end{aligned}
$$

and, by injectivity of the Laplace transform, $\mathcal{T}(t)\left(f_{n}\right)_{n \geq 1}=\left(T_{n}(t) f_{n}\right)_{n \geq 1}$. In particular, for all $f \in L,\left(T_{n}(t) f\right)_{n \geq 1}$ belongs to $\mathcal{L}$, i.e. $\lim _{n \rightarrow \infty} T_{n}(\bar{t}) f$ exists.

(The "only if" part) If for $f \in L,\left(T_{n}(t) f\right)_{n \geq 1}$ belongs to $\mathcal{L}$, then so does $\left(T_{n}(t) f_{n}\right)_{n \geq 1}$ for all $\left(f_{n}\right)_{n \geq 1} \in \mathcal{L}$. For $t \geq 0$ define $\mathcal{T}(t): \mathcal{L} \rightarrow \mathcal{L}$ and $T_{0}(t): L \rightarrow L$, by

$$
\mathcal{T}(t)\left(f_{n}\right)_{n \geq 1}=\left(T_{n}(t) f_{n}\right)_{n \geq 1}, \quad T_{0}(t) f=\lim _{n \rightarrow \infty} T_{n}(t) f .
$$

It is evident that $\mathcal{T}(t+s)=\mathcal{T}(t) \mathcal{T}(s)$ and $T_{0}(t+s)=T_{0}(t) T_{0}(s)$ for $s, t \geq 0$. Furthermore, $t \mapsto T_{0}(t) f$, being the pointwise limit of continuous, and thus measurable, functions, is itself strongly measurable ([32], p. 72, Th. 3.5.4). Therefore, by [32], p. 305, Th. 10.2.3, it is strongly continuous for $t>0$.

We now prove the same statement for $\{\mathcal{T}(t): t \geq 0\}$. Note that by the Pettis theorem ([32], p. 72, Th. 3.5.3) a Banach space valued function is strongly measurable iff it is weakly measurable and almost separably-valued. Fix $\left(f_{n}\right)_{n \geq 1} \in \mathcal{L}$ and let $f=\lim _{n \rightarrow \infty} f_{n}$. The vectors $T_{0}(w) f$ where $w$ is rational form a dense set in $Z=\left\{g \in L: g=T_{0}(t) f, t>0\right\}$. Let $\mathcal{Z}$ be the set of all sequences $\left(f_{n}\right)_{n \geq 1}$ which are ultimately constant of the form $T_{0}(w) f$ and initially of the form $f_{n}=T_{n}\left(w_{n}\right) f_{n}$, where the $w_{n}$ are rational.

Note that $\mathcal{Z}$ is countable and that its closure contains the set $\mathcal{W}=$ $\left\{\left(h_{n}\right)_{n \geq 1}:\left(h_{n}\right)_{n \geq 1}=\mathcal{T}(t)\left(f_{n}\right)_{n \geq 1}, t>0\right\}$. Indeed, for $t, \varepsilon>0$ there is $n_{0} \in \mathbb{N}$ such that for $n>n_{0},\left\|T_{n}(t) f_{n}-T_{0}(t) f\right\|<\varepsilon$. Consequently, there is a rational $w$ such that $\left\|T_{n}(t) f_{n}-T_{0}(w) f\right\|<\varepsilon$ for $n \geq n_{0}$, and since we may find rational numbers $w_{1}, \ldots, w_{n_{0}}$ such that $\left\|T_{n}(t) f_{n}-T_{n}\left(w_{n}\right) f_{n}\right\|<\varepsilon$ for $n \leq n_{0}$ one sees that $\widetilde{h}=\left(h_{n}\right)_{n \geq 1}$ where $h_{n}=T_{n}\left(w_{n}\right) f_{n}$ or $h_{n}=T_{0}(w) f$ if $n \leq n_{0}$ or $n>n_{0}$, respectively, belongs to $\mathcal{Z}$ and $\left\|\mathcal{T}(t)\left(f_{n}\right)_{n \geq 1}-\widetilde{h}\right\|<\varepsilon$, as desired.

Furthermore, $t \mapsto \mathcal{T}(t)$ is weakly measurable. Indeed, any functional $\Psi \in \mathcal{L}^{*}$ may be represented in the form $\Psi\left(f_{n}\right)_{n \geq 1}=\psi_{0}(f)+\sum_{n=1}^{\infty} \psi_{n}\left(f_{n}\right)$ where $f=\lim _{n \rightarrow \infty} f_{n}, \psi_{i} \in L^{*}$, and $\sum_{n=0}^{\infty}\left\|\psi_{n}\right\|_{L^{*}}<\infty$, which implies that $t \mapsto \Psi\left[\left(T_{n}(t) f_{n}\right)_{n \geq 1}\right]$ is a pointwise limit of measurable functions. Thus, we get the strong measurability of $\{\mathcal{T}(t): t \geq 0\}$ from Pettis's theorem. 
Finally,

$$
\begin{aligned}
\int_{0}^{\infty} e^{-\lambda t} \mathcal{T}(t)\left(f_{n}\right)_{n \geq 1} d t & =\left(\int_{0}^{\infty} e^{-\lambda t} T_{n}(t) f_{n} d t\right)_{n \geq 1}=\left(R_{\lambda, n} f_{n}\right)_{n \geq 1} \\
& =(\lambda-\mathcal{A})^{-1}\left(f_{n}\right)_{n \geq 1}
\end{aligned}
$$

and the proposition follows.

Corollary 1. For all $t>0$ and $\left(f_{n}\right)_{n \geq 1} \in \mathcal{L}$ we have $\mathcal{T}(t)\left(f_{n}\right)_{n \geq 1} \in \mathcal{L}^{\prime}$, and, consequently, $T_{0}(t) f \in L^{\prime}$ for all $f \in L$ and $t>0$.

Pr o of. This follows directly from (2.5) and Proposition 1.

Proposition 2. If the limit $\lim _{n \rightarrow \infty} T_{n}(t) f$ exists for all $f \in L$, then it is uniform in $t \in(0, \infty)$. Furthermore, for $f \in L^{\prime}$, the limit is almost uniform in $t \in[0, \infty)$.

Proof. The semigroup $\{\mathcal{T}(t): t \geq 0\}$, being measurable, is strongly continuous for $t>0$, and thus uniformly continuous on any compact subinterval of $(0, \infty)$. The same applies to $\left\{T_{0}(t): t \geq 0\right\}$. Fix $f \in L$ and $r>1$. For any $\varepsilon>0$, there exists a $\delta$ such that

$$
\left\|\mathcal{T}(t)(f)_{n \geq 1}-\mathcal{T}(s)(f)_{n \geq 1}\right\|<\varepsilon / 3, \quad\left\|T_{0}(t) f-T_{0}(s) f\right\|<\varepsilon / 3,
$$

whenever $|s-t|<\delta$ and $1 / r \leq s, t \leq r$. Therefore, for such $s, t$ and all $n \geq 1$ we get, recalling that $\mathcal{T}(t)\left(f_{n}\right)_{n \geq 1}=\left(T_{n}(t) f_{n}\right)_{n \geq 1}$,

$$
\begin{aligned}
\left\|T_{n}(t) f-T_{0}(t) f\right\| \leq & \left\|T_{n}(t) f-T_{n}(s) f\right\|+\left\|T_{n}(s) f-T_{0}(s) f\right\| \\
& +\left\|T_{0}(s) f-T_{0}(t) f\right\| \\
\leq & \frac{2}{3} \varepsilon+\left\|T_{n}(s) f-T_{0}(s) f\right\| .
\end{aligned}
$$

Set $s_{i}=1 / r+i \delta / 2, i=1, \ldots,[(2 / \delta)(r-1 / r)]$ where $[\cdot]$ is integer part. For any $t \in[1 / r, r]$, there exists an $i$ such that $\left|s_{i}-t\right|<\delta$. Since one can choose an $n_{0}$ such that $\sup _{i}\left\|T_{n}\left(s_{i}\right) f-T_{0}\left(s_{i}\right)\right\|<\varepsilon / 3$ for $n \geq n_{0}$, by (2.6) we get

$$
\sup _{t \in[1 / r, r]}\left\|T_{n}(t) f-T_{0}(t) f\right\|<\varepsilon \quad \text { for } n \geq n_{0},
$$

as desired.

Analogously, the last assertion of the proposition follows from the fact that the semigroups $\left\{\mathcal{T}(t)_{\mid \mathcal{L}^{\prime}}: t \geq 0\right\}$ and $\left\{T_{0}(t)_{\mid L^{\prime}}: t \geq 0\right\}$ are strongly continuous for $t \geq 0$.

Thus, in all the examples presented in [9-10], the limit is actually almost uniform in $t \in(0, \infty)$. In some cases, like the generalized telegraph equation with small parameter, this result is not easily seen from the original arguments as presented in [9]. 
Example 2. Suppose that $A$ is a closed operator such that

$$
(\lambda-A)^{-1} f=\int_{0}^{\infty} e^{-\lambda t} T(t) f d t \quad \text { for all } f \in L
$$

where $\{T(t): t \geq 0\}$ is a semigroup of equibounded operators, measurable yet in general discontinuous at 0 . Consider the Yosida approximation $e^{A_{\lambda} t}$ where $A_{\lambda}=\lambda^{2}(\lambda-A)^{-1}-\lambda$. Since the semigroup $\{T(t): t \geq 0\}$, being measurable, is strongly continuous for $t>0$ ([32], p. 305, Th. 10.2.3), it follows from [32], Th. 6.3 .3 (or [49]) that $\lim _{\lambda \rightarrow \infty} e^{A_{\lambda} t} f=T(t) f$, and the limit is almost uniform in $t \in(0, \infty)$.

A special case of a semigroup generated by a sectorial operator was considered in [10]; the proof was based on an approximation theorem for uniformly holomorphic semigroups given in [9] (see Proposition 5 below).

Proposition 3 (cf. [37], p. 359, Lemma 2.11). Suppose that (2.1) holds. The sequences $\left(T_{n}(t) f\right)_{n \geq 1}$ converge for all $f \in L$ iff the semigroups $\left\{T_{n}(t)\right.$ : $t \geq 0\}$ are uniformly Abel summable for $t>0$ in the sense that

$$
\begin{aligned}
& \lim _{\lambda \rightarrow \infty} \sup _{n \geq 1}\left\|T_{n}(t) \lambda\left(\lambda-A_{n}\right)^{-1} f-T_{n}(t) f\right\| \\
&=\lim _{\lambda \rightarrow \infty} \sup _{n \geq 1}\left\|\int_{0}^{\infty} \lambda e^{-\lambda s} T_{n}(t+s) f d s-T_{n}(t) f\right\|=0 .
\end{aligned}
$$

Proof. We first prove the "if" part. Let $\mathcal{A}$ be defined by (2.2). Since

$$
\left(T_{n}(t) \lambda\left(\lambda-A_{n}\right)^{-1} f\right)_{n \geq 1}=\mathcal{T}_{0}(t) \lambda \mathcal{R}_{\lambda}(f)_{n \geq 1}
$$

(see Theorem 1), (2.7) implies that the limit (2.4) exists for constant sequences. Furthermore, the sequences converging to 0 belong to $\mathcal{L}^{\prime}$ and thus satisfy an even stronger condition:

$$
\begin{aligned}
\lim _{\lambda \rightarrow \infty} \sup _{n \in \mathbb{N}} \| \lambda\left(\lambda-A_{n}\right)^{-1} & f_{n}-f_{n} \|_{L} \\
& =\lim _{\lambda \rightarrow \infty}\left\|\lambda(\lambda-\mathcal{A})^{-1}\left(f_{n}\right)_{n \geq 1}-\left(f_{n}\right)_{n \geq 1}\right\|_{\mathcal{L}}=0,
\end{aligned}
$$

which follows from the strong continuity of $\left\{\mathcal{T}_{0}(t): t \geq 0\right\}$ on $\mathcal{L}^{\prime}$. Therefore, (ii) of Theorem 1 is satisfied because the constant sequences and sequences converging to 0 form a dense subset of $\mathcal{L}$. Theorem 1 and Proposition 1 complete the proof of the "if" part.

Conversely, if $\left(T_{n}(t) f\right)_{n>1}$ converges for $t>0$ and $f \in L$, then, by Proposition 1 , the family $\{\mathcal{T}(t): t \geq 0\}$ where $\mathcal{T}(t)\left(f_{n}\right)_{n \geq 1}=\left(T_{n}(t) f_{n}\right)_{n \geq 1}$ is the semigroup generated by the operator $\mathcal{A}$ in the sense of Theorem 1 . Applying (iii) to a constant sequence $\widetilde{f}=\left(f_{n}\right)_{n \geq 1}=(f)_{n \geq 1}$ we get (2.7). Indeed, for all $n, T_{n}(t) \lambda\left(\lambda-A_{n}\right)^{-1} f \rightarrow T_{n}(t) f$ as $\lambda \rightarrow \infty$, and, by (2.8), the existence of the limit (2.4) (in the space $\mathcal{L}$ equipped with the supremum norm) implies that the convergence is uniform in $n \in \mathbb{N}$. 
Note that if (2.7) holds for any fixed $t_{0}>0$ then it holds for all $t>t_{0}$, but not necessarily for $t<t_{0}$. This explains to some extent how discontinuity at $t=0$ of the limit semigroup comes into existence.

A few remarks on differentiable semigroups are now in order since our next proposition is devoted to the convergence of equidifferentiable semigroups. Recall that the semigroup $\{\mathcal{T}(t): t \geq 0\}$ generated by an operator $A$ is said to be differentiable iff $\mathcal{T}(t) f \in \mathcal{D}(A)$ for all $f \in \mathcal{L}$ and $t>0$. Differentiable semigroups were studied e.g. by Hille [31], Yosida [54], and Pazy [47] (see also [48], p. 57, Th. 4.8), who gave necessary and sufficient conditions for an operator $A$ to generate a differentiable semigroup.

Furthermore, there exists a close connection between differentiable semigroups and the well-known class of analytic semigroups: any holomorphic (analytic) semigroup is differentiable, and a differentiable semigroup is analytic iff there exists a constant $C>0$ such that $\|A T(t)\| \leq C / t$ for all $t>0$ ([48], p. 61, Th. 5.2). Let us also mention the recent paper by M. Renardy [50] who presented an interesting example showing that the class of differentiable semigroups is not stable under bounded perturbations. The paper $[21]$ is devoted to the same subject.

The necessary and sufficient conditions for an operator $A$ to generate a differentiable semigroup, due to A. Pazy, are presented in the following theorem.

Theorem 2. Let $\mathcal{A}$ be the infinitesimal generator of a strongly continuous semigroup $\left\{\mathcal{T}_{0}(t): t \geq 0\right\}$ satisfying $\left\|\mathcal{T}_{0}(t)\right\| \leq M$. The semigroup is differentiable iff for all $b>0$ there exist constants $a_{b} \in \mathbb{R}$ and $C_{b}>0$ such that

$$
\varrho(\mathcal{A}) \supset \Sigma_{b}:=\left\{\lambda: \operatorname{Re} \lambda>a_{b}-b \log |\operatorname{Im} \lambda|\right\}
$$

and

$$
\left\|\mathcal{R}_{\lambda}(\mathcal{A})\right\| \leq C_{b}|\operatorname{Im} \lambda| \quad \text { for } \lambda \in \Sigma_{b}, \operatorname{Re} \lambda \leq 0
$$

Corollary 2. Let $\mathcal{A}$ satisfy (2.3). If the semigroup $\left\{\mathcal{T}_{0}(t): t \geq 0\right\}$ generated by the operator $\mathcal{A}_{0}$ is differentiable then the semigroup $\{\mathcal{T}(t): t \geq 0\}$ described in statement (i) of Theorem 1 exists.

P r o of. Since Range $\mathcal{R}_{\lambda}(\mathcal{A}) \subset \mathcal{L}^{\prime}$, it is obvious that our assumptions imply statement (iv) of Theorem 1, and the corollary follows.

Proposition 4. If the semigroups $\left\{T_{n}(t): t \geq 0\right\}$ are equidifferentiable in the sense that for all $b>0$ there exist constants $a_{b} \in \mathbb{R}$ and $C_{b}>0$ such that for all $n \in \mathbb{N}$,

$$
\varrho\left(A_{n}\right) \supset \Sigma_{b}:=\left\{\lambda: \operatorname{Re} \lambda>a_{b}-b \log |\operatorname{Im} \lambda|\right\}
$$


and

$$
\left\|R_{\lambda, n}\right\| \leq C_{b}|\operatorname{Im} \lambda| \quad \text { for } \lambda \in \Sigma_{b}, \operatorname{Re} \lambda \leq 0, n \geq 1,
$$

then (2.1) holds iff $\lim _{n \rightarrow \infty} T_{n}(t) f$ exists for all $t>0$ and $f \in L$. Furthermore, for $f \in L^{\prime}=\overline{\text { Range } R_{\lambda}}$ the limit is almost uniform in $t \in[0, \infty)$.

Proof. From $R_{\lambda, n} f=\int_{0}^{\infty} e^{-\lambda t} T_{n}(t) f d t, \operatorname{Re} \lambda>0, f \in L$, it is evident that (2.1) is necessary for the convergence of $T_{n}(t)$.

Conversely, suppose that (2.1) holds. The "only if" part consists of 3 steps.

SteP 1. Fix $b>0$. Consider a purely imaginary $\lambda_{0} \in \Sigma_{b}$. We shall prove that $\lim _{n \rightarrow \infty} R_{\lambda_{0}, n} f$ exists for all $f \in L$, and thus $\lambda_{0} \in \varrho(\mathcal{A})$ where $\mathcal{A}$ is defined by $(2.2)$. Setting $s=1 /\left(4\left|\operatorname{Im} \lambda_{0}\right| C_{b}\right)$ and $\lambda_{1}=\lambda_{0}+s$ yields, by $(2.11)$,

$$
R_{\lambda_{1}, n}=\sum_{k=0}^{\infty}(-s)^{k} R_{\lambda_{0}, n}^{k+1}, \quad\left\|R_{\lambda_{1}, n}\right\| \leq\left\|R_{\lambda_{0}, n}\right\| \sum_{k=0}^{\infty} \frac{1}{4^{k}} \leq \frac{4}{3}\left|\operatorname{Im} \lambda_{0}\right| C_{b} .
$$

Thus, $R_{\lambda_{0}, n}=\sum_{k=0}^{\infty} s^{k} R_{\lambda_{1}, n}^{k+1}$, the series $\sum_{k=0}^{\infty}(1 / 3)^{k}$ being a majorant to the series on the right-hand side. This implies the convergence of $R_{\lambda_{0}, n}$, since $\operatorname{Re} \lambda_{1}>0$.

Accordingly, setting $\mathcal{R}_{\lambda_{0}}\left(f_{n}\right)_{n \geq 1}=\left(R_{\lambda_{0}, n} f_{n}\right)_{n \geq 1}$ we get a bounded operator in $\mathcal{L}$, with $\left\|\mathcal{R}_{\lambda_{0}}\right\| \leq C_{b}\left|\operatorname{Im} \lambda_{0}\right|$, which is both the right and left inverse of $\lambda_{0}-\mathcal{A}$.

STEP 2. Keep $b>0$ fixed. Let $S_{b}=\Sigma_{b} \cap\{\lambda: \operatorname{Re} \lambda \leq 0\}$. Since $\varrho(\mathcal{A})$ is open in $C, S_{b}^{\prime}=S_{b} \cap \varrho(\mathcal{A})$ is open in $S_{b}$. We now show that $S_{b}^{\prime}=S_{b}$. Since $S_{b}$ is connected and $S_{b}^{\prime}$ is non-empty (by Step 1), it is enough to prove that $S_{b}^{\prime}$ is closed in $S_{b}$. This is actually a classical argument (see e.g. [48], p. 87, [23], p. 12, Lemma 2.3).

For $\lambda \in S_{b}$ and $\left(f_{n}\right)_{n \geq 1} \in \mathcal{D}(\mathcal{A})$,

$$
\left\|(\lambda-\mathcal{A})\left(f_{n}\right)_{n \geq 1}\right\|_{\mathcal{L}}=\sup _{n \in \mathbb{N}}\left\|\left(\lambda-A_{n}\right) f_{n}\right\|_{L} \geq \frac{1}{C_{b}|\operatorname{Im} \lambda|}\left\|\left(f_{n}\right)_{n \geq 1}\right\|_{\mathcal{L}} .
$$

Since $\mathcal{A}$ is closed, the set Range $(\lambda-\mathcal{A}$ ) is closed (see e.g. [23], p. 11, Lemma 2.2). Suppose $\lambda_{n} \rightarrow \lambda, \lambda_{n} \in S_{n}^{\prime}, \lambda \in S_{b}$. We need to prove that $\lambda \in \varrho(\mathcal{A})$. By (2.12) it is enough to show that $\operatorname{Range}(\lambda-\mathcal{A})$ is dense in $\mathcal{L}$ and, thus, it equals $\mathcal{L}$.

For $\widetilde{g} \in \mathcal{L}$ put $\widetilde{g}_{n}=\left(\lambda_{n}-\mathcal{A}\right)^{-1} \widetilde{g}$ to obtain (cf. [23], p. 12)

$$
\left\|\widetilde{g}-(\lambda-\mathcal{A}) \widetilde{g}_{n}\right\|=\left\|\left(\lambda-\lambda_{n}\right) \widetilde{g}_{n}\right\| \leq C_{b}\left|\operatorname{Im} \lambda_{n}\right| \cdot\left|\lambda-\lambda_{n}\right| \cdot\|\widetilde{g}\| \underset{n \rightarrow \infty}{\longrightarrow} 0,
$$

which completes the proof.

SteP 3. By Steps 1 and $2, S_{b} \subset \varrho(\mathcal{A})$ for all $b>0$ and

$$
\left\|(\lambda-\mathcal{A})^{-1}\right\| \leq C_{b}|\operatorname{Im} \lambda| .
$$


Since the operator $\mathcal{A}_{0}$, the part of $\mathcal{A}$ in $\mathcal{L}^{\prime}=\overline{\mathcal{D}(\mathcal{A})}$, satisfies $\left(\lambda-\mathcal{A}_{0}\right)^{-1}=$ $(\lambda-\mathcal{A})_{\mid \mathcal{L}^{\prime}}^{-1}$, Theorem 2 and the estimate $(2.14)$ prove that the semigroup generated by $\mathcal{A}_{0}$ is differentiable. By Corollary 2, for the operator $\mathcal{A}$ defined by (2.2) the semigroup described in Theorem 1(i) exists. Proposition 1 completes the proof.

Proposition 5 ([9], Proposition 4). Let $\left\{T_{n}(t): t \geq 0\right\}$ be strongly continuous equibounded semigroups with $\left\|T_{n}(t)\right\| \leq M$. Suppose that they are uniformly holomorphic, i.e. the resolvent sets of the corresponding infinitesimal generators contain not only the half plane $\operatorname{Re} \lambda>0$ but also a sector $|\arg \lambda|<\pi / 2+\omega$ where $\omega>0$, and that, for any $\delta>0$,

$$
\left\|\left(\lambda-A_{n}\right)^{-1}\right\| \leq M_{\delta} /|\lambda| \quad \text { for }|\arg \lambda|<\pi / 2+\omega-\delta,
$$

with $\omega$ independent of $n$ and $M_{\delta}$ independent of $\lambda$. Then $T_{n}(t)$ converges for $f \in L$ and $t>0$ iff (2.1) holds.

Proof. The necessity of (2.1) is proven as in Proposition 2. The sufficiency argument also follows the lines of Proposition 2: for fixed $\delta>0$ the set $S_{\delta}^{\prime}=S_{\delta} \cap \varrho(\mathcal{A})$ where $S_{\delta}=\{\lambda:|\arg \lambda|<\pi / 2+\omega-\delta\}$ is both closed and open in $S_{\delta}$ (cf. (2.13) - replace $C_{b}\left|\operatorname{Im} \lambda_{n}\right|$ by $\left.M_{\delta} /\left|\lambda_{n}\right|\right)$. Condition (2.1) implies that $S_{\delta}^{\prime}$ is non-empty and thus equals $S_{\delta}$. Consequently, $S_{\delta} \subset \varrho(\mathcal{A})$ and

$\left\|(\lambda-\mathcal{A})^{-1}\left(f_{n}\right)_{n \geq 1}\right\|=\left\|\left(\left(\lambda-A_{n}\right)^{-1} f_{n}\right)_{n \geq 1}\right\| \leq \frac{M_{\delta}}{\left|\lambda_{n}\right|}\left\|\left(f_{n}\right)_{n \geq 1}\right\| \quad$ for $\lambda \in S_{\delta}$.

The semigroup generated by $\mathcal{A}_{0}$ is therefore holomorphic (see e.g. [34], p. 488, or [48], p. 60) and, in particular, differentiable. Thus, the arguments at the end of Proposition 4 can be applied.

REMARK 1. One can prove the above proposition by using the explicit formula for $\left\{T_{n}(t): t \geq 0\right\}$, i.e. by employing the Dunford integral - this was done in [9]. One may also obtain it as a corollary of Proposition 4 by proving that uniformly holomorphic semigroups are equidifferentiable. Note, however, that while the last idea seems to be natural, the calculations it leads to, though straightforward, are rather long.

3. Convergence theorems and asymptotic analysis. In this section we study the asymptotic behaviour of semigroups, as viewed from the standpoint of the related approximation theory briefly presented in the foregoing part of the paper. First of all, note that in dealing with the asymptotic behaviour of a semigroup $\{T(t): t \geq 0\}$ of equibounded operators it seems natural to use the sequence $\left\{T_{n}(t): t \geq 0\right\}$ of semigroups defined by

$$
T_{n}(t)=T(n t)
$$


as a tool allowing the use of approximation theorems for semigroups. However, this idea fails to be fruitful if we restrict ourselves to applications of the Trotter-Kato-Neveu-Kurtz theorem. (We add Kurtz's name here since he was, to the best of our knowledge, the first to mention the effect of the convergence of the integrals $\int_{0}^{t} T_{n}(s) f d s$ even when the semigroups themselves do not converge, as described in Theorem 3 below-see [38], p. 29.) The reason is that, as will become clear from the proof presented below, the Trotter-Kato-Neveu-Kurtz theorem may be viewed as a special case of Proposition 6 (below) plus a density argument leading to the convergence of the semigroups themselves; Proposition 6 states that the Laplace transforms of a sequence of equibounded functions converge iff the functions converge in the mean. In the case of the semigroups (3.1) the density argument works only for the kernel of the generator of the semigroup $\{T(t): t \geq 0\}$ and is of little use. Therefore, the Trotter-Kato-Neveu-Kurtz theorem leads only to mean ergodic theorems.

On the other hand, the theorems presented in the preceding section turn out to be of different nature and when applying them we can use the semigroups (3.1).

Proposition 6. Let $F_{n}(t)$ be equibounded measurable functions defined on $[0, \infty)$ with values in a Banach space $L$. The following are equivalent:

(i) $\lim _{n \rightarrow \infty} \int_{0}^{\infty} e^{-\lambda t} F_{n}(t) d t$ exists for all $\lambda>0$,

(ii) $\lim _{n \rightarrow \infty} \int_{0}^{t} F_{n}(s) d s$ exists for all $t>0$,

(iii) $\lim _{n \rightarrow \infty} \int_{0}^{\infty} \phi(t) F_{n}(t) d t$ exists for all $\phi \in \mathcal{L}^{1}\left(\mathbb{R}^{+}\right)$.

Proof. Since $e_{\lambda}(s):=e^{-\lambda s}(\lambda>0)$ and $1_{[0, t)}(t>0)$ form total subsets of $\mathcal{L}^{1}\left(\mathbb{R}^{+}\right)$, all the conditions above are equivalent to the strong convergence of the equibounded operators $S_{n}: \mathcal{L}^{1}\left(\mathbb{R}^{+}\right) \rightarrow L$ where $S_{n} \phi=$ $\int_{0}^{\infty} \phi(t) F_{n}(t) d t,\left\|S_{n}\right\| \leq \operatorname{ess} \sup \left\|F_{n}(t)\right\|$.

REMARK 2. Since $\lim _{n \rightarrow \infty} \int_{0}^{t} F_{n}(s) d s$ are Lipschitz continuous with the same constant the limit in (ii) is almost uniform in $[0, \infty)$ and the limit function is Lipschitz continuous as well.

Both the above proposition and its proof are actually special cases of [29], pp. 166-168. Theorems of this type are generally ascribed to C. Lizama [40], yet Lizama's Theorem 1.2 is a special case of Lemma 2.11 in the wellknown paper by T. G. Kurtz [37] (if the boundedness assumption in the lemma is replaced by that of exponential growth, which is of no importance for the proof; see also [14], Proposition 1, for a more handy version). It is also interesting to compare Kurtz's result mentioned in the introduction ([38], p. 29, Proposition 2.22) with Arendt's theorem on generation of onceintegrated Lipschitz continuous semigroups (cf. [11]). 
Theorem 3. Let $\left\{T_{n}(t): t \geq 0\right\}$ be equibounded semigroups. For fixed $f \in L$, the following conditions are equivalent:

(i) $\lim _{n \rightarrow \infty}\left(\lambda-A_{n}\right)^{-1} f=: R_{\lambda} f$ exists for all $\lambda>0$,

(ii) $\lim _{n \rightarrow \infty} \int_{0}^{\infty} \phi(t) T_{n}(t) f d t=: T(\phi) f$ exists for all $\phi \in \mathcal{L}^{1}\left(\mathbb{R}^{+}\right)$,

(iii) $\lim _{n \rightarrow \infty} \int_{0}^{t_{0}} T_{n}(t) f d t=: T\left(1_{\left[0, t_{0}\right)}\right) f$ exists for all $t_{0}>0$.

Furthermore, the limit $\lim _{n \rightarrow \infty} T_{n}(t) f$ exists almost uniformly in $[0, \infty)$ iff $f \in L^{\prime}:=\overline{\mathcal{R}}$ where $\mathcal{R}=\{f \in L: f=T(\phi) g$ for some $g \in L$ and $\phi \in$ $\left.\mathcal{L}^{1}\left(\mathbb{R}^{+}\right)\right\}$.

P r o of. Conditions (i) through (iii) are equivalent by Proposition 6 (put $\left.F_{n}(t)=T_{n}(t) f\right)$.

Moreover, the semigroup of right translations in $\mathcal{L}^{1}\left(\mathbb{R}^{+}\right)$is of class $C_{0}$ and, thus, for fixed $\phi \in \mathcal{L}^{1}\left(\mathbb{R}^{+}\right)$, the function $[0, \infty) \ni t \mapsto \phi_{t} \in \mathcal{L}^{1}\left(\mathbb{R}^{+}\right)$ where $\phi_{t}(x)=\phi(x-t)$ for $x>t$ and 0 elsewhere, is continuous. Suppose $f=T(\phi) g$. Put $g_{n}=\int_{0}^{\infty} \phi(t) T_{n}(t) g d t$. We have

and

$$
T_{n}(t) g_{n}=\int_{0}^{\infty} \phi(s) T_{n}(t+s) g d s=\int_{0}^{\infty} \phi_{t}(s) T_{n}(s) g d s=T_{n}\left(\phi_{t}\right) g,
$$

$$
\begin{aligned}
\sup _{t \in\left[0, t_{0}\right]} \| T_{n}(t) & g-T\left(\phi_{t}\right) g \| \\
\leq & \sup _{t \in\left[0, t_{0}\right]}\left\|T_{n}(t) g-T_{n}(t) g_{n}\right\|+\sup _{t \in\left[0, t_{0}\right]}\left\|T_{n}(t) g_{n}-T\left(\phi_{t}\right) g\right\| \\
\leq & M\left\|g-g_{n}\right\|+\sup _{t \in\left[0, t_{0}\right]}\left\|T_{n}\left(\phi_{t}\right) g-T\left(\phi_{t}\right) g\right\| .
\end{aligned}
$$

The last expression tends to 0 , since $T_{n} g \rightarrow T g$ and the $\phi_{t} \in \mathcal{L}^{1}\left(\mathbb{R}^{+}\right)$ $\left(t \in\left[0, t_{0}\right]\right)$ form a compact set. By a continuity argument, the convergence holds on the whole of $L^{\prime}$.

Conversely, if $\lim _{n \rightarrow \infty} T_{n}(t) f=: u(t)$ exists almost uniformly in $t \in$ $[0, \infty)$, then $u$ is continuous, $u(0)=f$, and

$$
\begin{aligned}
u(0) & =\lim _{t \rightarrow 0} \frac{1}{t} \int_{0}^{t} u(s) d s=\lim _{t \rightarrow 0} \frac{1}{t} \lim _{n \rightarrow \infty} \int_{0}^{t} T_{n}(s) f d s \\
& =\lim _{t \rightarrow 0} \frac{1}{t} \lim _{n \rightarrow \infty} T_{n}\left(1_{[0, t)}\right) f=\lim _{t \rightarrow 0} T\left(\frac{1}{t} 1_{[0, t)}\right) f,
\end{aligned}
$$

which completes the proof.

REMARK 3. One can show that $\phi \mapsto T(\phi)$ is a representation of the convolution algebra $\mathcal{L}^{1}\left(\mathbb{R}^{+}\right)$in the algebra of bounded operators on the Banach space which consists of those $f \in L$ for which the limits (ii) above exist. Therefore, according to the theorem due to Hewitt et al. [17-18, 28, 
30], $\mathcal{R}=\overline{\mathcal{R}}$, and the density argument used at the end of the proof of Theorem 3 is redundant. We have avoided using the result mentioned above to make the proof as elementary as possible.

Proposition 6 also yields a simple mean ergodic theorem which we now prove.

Proposition 7. Suppose $F:[0, \infty) \rightarrow L$ is bounded and measurable. The following are equivalent:

(i) $\lim _{\lambda \rightarrow 0} \lambda \int_{0}^{\infty} e^{-\lambda t} F(t) d t$ exists,

(ii) $\lim _{t \rightarrow \infty} \frac{1}{t} \int_{0}^{t} F(s) d s$ exists,

(iii) $\lim _{\lambda \rightarrow 0} \lambda \int_{0}^{\infty} \phi(\lambda t) F(t) d t$ exists for all $\phi \in \mathcal{L}^{1}\left(\mathbb{R}^{+}\right)$.

Proof. One may apply Proposition 6 directly to $F_{n}(t)=F\left(a_{n} t\right)$ where $a_{n} \rightarrow \infty$ and establish equivalence of (i)-(iii) by straightforward calculations. Let us, however, quote Davies ([20], p. 123): "Equivalence of (i) and (ii) is established by noting that each implies that $\lim _{\lambda \rightarrow 0} \lambda \int_{0}^{\infty} \phi(\lambda t) F(t) d t$ exists for all $\phi \in \mathcal{L}^{1}\left(\mathbb{R}^{+}\right)$, by density argument" (cf. the proof of Proposition 6).

EXAMPLE 3 ([20], p. 123). If $\{T(t): t \geq 0\}$ is a semigroup of equibounded operators, then, for every $f \in L$, the function $F(t)=T(t) f$ is bounded and measurable, and therefore, the $\operatorname{limit}_{\lambda \rightarrow 0} \lambda \int_{0}^{\infty} e^{-\lambda t} T(t) f d t$ exists iff $\lim _{t \rightarrow \infty} \frac{1}{t} \int_{0}^{t} T(s) f d s$ does.

For a discussion of convergence rates in ergodic theorems for semigroups see $[25]$.

ExAmple 4 (cf. [22]). Suppose $T$ is a power-bounded operator in $L$ (i.e. $\left.\sup _{n \in \mathbb{N}}\left\|T^{n}\right\|<\infty\right)$, and define the $\mathcal{L}(L, L)$-valued function by $F(t)=T^{[t]}$ (see [6], p. 22) where [.] is integer part. The function $F$ is bounded and

$$
\begin{aligned}
\lambda \int_{0}^{\infty} e^{-\lambda t} F(t) d t & =\lambda \sum_{n=0}^{\infty} T^{n} \int_{n}^{n+1} e^{-\lambda t} d t \\
& =\left(1-e^{-\lambda}\right) \sum_{n=0}^{\infty}\left(e^{-\lambda} T\right)^{n}=\left(e^{\lambda}-1\right)\left(e^{\lambda}-T\right)^{-1}
\end{aligned}
$$

and

$$
\begin{aligned}
\frac{1}{t} \int_{0}^{t} F(s) d s & =\frac{1}{t} \int_{0}^{[t]} F(s) d s+\frac{1}{t} \int_{[t]}^{t} F(s) d s \\
& =\frac{[t]}{t} \frac{I+T+\ldots+T^{[t]-1}}{[t]}+\frac{t-[t]}{t} T^{[t]} .
\end{aligned}
$$


This proves, by Proposition 7 , that $\lim _{n \rightarrow \infty}\left(I+T+\ldots+T^{n-1}\right) / n$ exists iff $\lim _{\lambda \rightarrow 1}(\lambda-1)(\lambda-T)^{-1}$ exists (both limits in the $\mathcal{L}(L, L)$ norm). A parallel result in the strong topology is established in the same way. The condition corresponding to (iii) of Proposition 7 is that the limit

$$
\lim _{\lambda \rightarrow 0} \lambda \sum_{n=0}^{\infty} T^{n} \int_{n}^{n+1} \phi(\lambda t) d t=\lim _{\lambda \rightarrow 0} \sum_{n=0}^{\infty} T^{n} \int_{\lambda n}^{\lambda(n+1)} \phi(t) d t
$$

exists for all $\phi \in \mathcal{L}^{1}\left(\mathbb{R}^{+}\right)$.

Now, let $\{T(t): t \geq 0\}$ be a continuous semigroup of equibounded operators with infinitesimal generator $A$. Define the semigroups $\left\{T_{n}(t): t \geq 0\right\}$ by (3.1). The corresponding infinitesimal generators $A_{n}$ and resolvents are given by

$$
A_{n}=n A, \quad\left(\lambda-A_{n}\right)^{-1}=\frac{1}{n}\left(\frac{\lambda}{n}-A\right)^{-1} .
$$

Lemma 1 (cf. [20], Th. 5.1, p. 123). Under the above assumptions and notations, the subspace $L^{\prime}$ defined in Theorem 3 equals $\operatorname{Ker} A$.

Proof. If $f=T\left(e_{\lambda}\right) g$, where $e_{\lambda}(t)=e^{-\lambda t}, \lambda>0, g \in L$, then

$$
f=\lim _{n \rightarrow \infty} \frac{1}{n}\left(\frac{\lambda}{n}-A\right)^{-1} g .
$$

The operator $A$ is closed. Thus, since $g_{n}:=\frac{1}{n}\left(\frac{\lambda}{n}-A\right)^{-1}$ belongs to $\mathcal{D}(A)$ and

$$
A g_{n}=\frac{\lambda}{n} g_{n}-\frac{1}{n} g \rightarrow 0 \quad \text { as } n \rightarrow \infty,
$$

it follows that $f \in \mathcal{D}(A)$ and $A f=0$; to prove that $\operatorname{Ker} A \subset L^{\prime}$ is equally simple. Finally, since Ker $A$ is closed and $L^{\prime}$ is the closure of the linear span of $\left\{f: f=T\left(e_{\lambda}\right) g\right\}$, the proof is complete.

Proposition 8. For fixed $f \in L$, the following limits exist simultaneously:

(i) $\lim _{t \rightarrow \infty} \frac{1}{t} \int_{0}^{t} T(s) f d s=g$,

(ii) $\lim _{n \rightarrow \infty} \frac{1}{t} \int_{0}^{t} T_{n}(s) f d s=h($ for all $t>0)$,

(iii) $\lim _{n \rightarrow \infty} \frac{1}{n}\left(\frac{1}{n}-A\right)^{-1} f$.

Furthermore, $g=h$. 
P r o o f. For (ii) and (iii) the conclusion follows from (3.3) and Theorem 3. Let (i) exist. Then, for each $t>0$,

$$
\frac{1}{t} \int_{0}^{t} T_{n}(s) f d s=\frac{1}{t} \int_{0}^{t} T(n s) f d s=\frac{1}{n t} \int_{0}^{n t} T(u) f d u \underset{n \rightarrow \infty}{\longrightarrow} g .
$$

Conversely, let (ii) exist and let $t=[t]+t^{\prime}$. We have

$$
\frac{1}{t} \int_{0}^{t} T(s) f d s=\frac{[t]}{t} \frac{1}{[t]} \int_{0}^{[t]} T(s) f d s+\frac{1}{t} \int_{[t]}^{t} T(s) f d s ;
$$

thus

$$
\begin{aligned}
\left\|\frac{1}{t} \int_{0}^{t} T(s) f d s-\lim _{n \rightarrow \infty} \int_{0}^{1} T_{n}(s) f d s\right\| \\
\leq\left\|\frac{[t]}{t} \frac{1}{[t]} \int_{0}^{[t]} T(s) f d s-h\right\|+\frac{t-[t]}{t} M\|f\| \underset{t \rightarrow \infty}{\longrightarrow} 0 .
\end{aligned}
$$

Our results show that concerning the existence of the $\operatorname{limit}_{\lim } \lim _{n \rightarrow \infty} T(t) f$, all we can get using (3.1) and the Trotter-Kato-Neveu-Kurtz theorem is Proposition 8 above; indeed, by Lemma 1 , in this case $L^{\prime}=\operatorname{Ker} A$, and therefore besides the convergence of the averages $\frac{1}{t} \int_{0}^{t} T(s) f d s$ one obtains merely the trivial information that $\lim _{n \rightarrow \infty} T(t) f$ exists when $f$ is a fixed point of the semigroup. We have, however, the following proposition.

Proposition 9. Let $\{T(t): t \geq 0\}$ be a semigroup of equibounded operators generated by an operator $A$. The following two statements are equivalent:

(i) for all $f \in L$ the limit $\lim _{t \rightarrow \infty} T(t) f$ exists,

(ii) the semigroups $\left\{T_{n}(t): t \geq 0\right\}$, where $T_{n}(t)=T(n t)$, converge on $L$ almost uniformly in $t \in(0, \infty)$.

Furthermore, if (i) or (ii) holds, then there exists a projection $P: L \rightarrow$ $L^{\prime}=\operatorname{Ker} A$ (cf. [20], p. 123, Th. 5.1, [23], p. 39, Lemma 7.3), and the semigroup $T_{0}(t) f:=\lim _{n \rightarrow \infty} T_{n}(t) f$ is given by $T_{0}(t) f=P f$.

Proof. Since (i) $\Rightarrow$ (ii) is immediate it is enough to prove the converse.

By Lemma $1, L^{\prime}=\operatorname{Ker} A$. It is evident that for all $f \in L^{\prime}, T(t) f=f$ and $T_{0}(t) f=f$. Take $t_{0}>0$ and let $t=n t_{0}+t^{\prime}$ where $0 \leq t^{\prime}<t_{0}$. Since, by Corollary $1, T_{0}\left(t_{0}\right) f$ belongs to $L^{\prime}$, we get

$$
\begin{aligned}
\left\|T(t) f-T_{0}\left(t_{0}\right) f\right\| & =\left\|T\left(t^{\prime}\right) T\left(n t_{0}\right) f-T\left(t^{\prime}\right) T_{0}\left(t_{0}\right) f\right\| \\
& \leq \mathrm{const} \cdot\left\|T\left(n t_{0}\right) f-T_{0}\left(t_{0}\right) f\right\| \rightarrow 0 \quad \text { as } n \rightarrow \infty,
\end{aligned}
$$


and (i) follows. The operator $P$ is given by

$$
P f=\lim _{t \rightarrow \infty} T(t) f=\lim _{n \rightarrow \infty} T_{n}\left(t_{0}\right) f=T_{0}\left(t_{0}\right) f .
$$

Note that the limit (i) is actually uniform in any interval $[r, \infty)$ where $r>0$.

As seen from Proposition 8, a necessary condition for the existence of the limit in Proposition 9(i) is the existence of $\lim _{n \rightarrow \infty} \frac{1}{n}\left(\frac{1}{n}-A\right)^{-1} f$. As an application of Proposition 5 we now show that the converse also holds provided $\{T(t): t \geq 0\}$ is holomorphic.

Proposition 10. Suppose $\{T(t): t \geq 0\}$ is a holomorphic semigroup of equibounded operators generated by an operator $A$. The following statements are equivalent:

(i) $\lim _{t \rightarrow \infty} T(t) f$ exists for all $f \in L$,

(ii) $\lim _{n \rightarrow \infty} \frac{1}{n}\left(\frac{1}{n}-A\right)^{-1} f$ exists for all $f \in L$,

(iii) $\lim _{\varepsilon \rightarrow 0} \varepsilon(\varepsilon-A)^{-1} f$ exists for all $f \in L$.

Furthermore, $g \in L$ may be obtained as one of the limits above iff $g$ belongs to $\operatorname{Ker} A$.

Proof. Suppose (i) holds. Fix a sequence $a_{n} \rightarrow \infty$ and set $T_{n}(t)=$ $T\left(a_{n} t\right)$. Of course, $\left\{T_{n}(t): t \geq 0\right\}$ converge and thus for any $\lambda>0$ the limit

$$
\lim _{n \rightarrow \infty}\left(\lambda-a_{n} A\right)^{-1} f=\lim _{n \rightarrow \infty} \frac{1}{a_{n}}\left(\frac{\lambda}{a_{n}}-A\right)^{-1} f
$$

exists. Setting $\lambda=1$ and $b_{n}=1 / a_{n}$ we see that for any $b_{n} \rightarrow 0\left(b_{n} \neq 0\right)$ the $\operatorname{limit}_{\lim } \rightarrow \infty b_{n}\left(b_{n}-A\right)^{-1} f$ exists, i.e. we have (iii).

Clearly, (iii) implies (ii).

Finally, suppose that (ii) holds. Since

$$
\frac{1}{n}\left(\frac{1}{n}-A\right)^{-1}=(1-n A)^{-1}=R_{1}\left(A_{n}\right)
$$

where $A_{n}=n A$ it follows that $\lim _{n \rightarrow \infty} R_{\lambda}\left(A_{n}\right)$ exists for all $\lambda>0$ and $f \in L$. The semigroups $\left\{T_{n}(t): t \geq 0\right\}$ generated by $A_{n}$ are uniformly holomorphic; indeed, there exist positive constants $M$ and $\omega$ such that $S_{\omega}:=$ $\{z \in \mathbb{C}:|\arg z| \leq \pi / 2+\omega\} \subset \varrho(A)$ and $\left\|R_{\lambda}(A)\right\| \leq M /|\lambda|$ for $\lambda \in S_{\omega} ;$ therefore, if $\lambda \in S_{\omega}$ then $\lambda / n$ also belongs to $S_{\omega}$, and

$$
\left\|R_{\lambda}\left(A_{n}\right)\right\|=\left\|(\lambda-n A)^{-1}\right\|=\frac{1}{n}\left\|\left(\frac{\lambda}{n}-A\right)^{-1}\right\| \leq \frac{\frac{1}{n} M}{\left|\frac{\lambda}{n}\right|}=\frac{M}{|\lambda|} .
$$

By Proposition 5 the $\operatorname{limit}_{\lim } \lim _{n} T_{n}(t) f=\lim _{n \rightarrow \infty} T(n t) f$ exists. Proposition 9 completes the proof. 
Example 3. Consider the space $L=C_{[0, \infty]}$ of all continuous functions $f:[0, \infty) \rightarrow C$ with a finite limit at infinity, equipped with the norm $\|f\|=\sup _{x>0}|f(x)|$. Given $\varepsilon>0$, define the domain of an operator $A_{\varepsilon}$ as the set of all twice continuously differentiable functions $f$ with $f^{\prime \prime} \in L$ which satisfy $f(0)=\varepsilon f^{\prime}(0)$, and set $A_{\varepsilon} f=\frac{1}{2} f^{\prime \prime}$. For every $\varepsilon>0$, the operator $A_{\varepsilon}$ is the infinitesimal generator of a positive and holomorphic contraction semigroup $\left\{S_{\varepsilon}(t): t \geq 0\right\}$ acting in $L$, and, furthermore ([9], (2.26)),

$$
\begin{aligned}
(\lambda & \left.-A_{\varepsilon}\right)^{-1} g(x) \\
& =\frac{1}{\sqrt{2 \lambda}} \int_{0}^{\infty} e^{-\sqrt{2 \lambda}|x-y|} g(y) d y+H(\varepsilon \sqrt{2 \lambda}) \frac{1}{\sqrt{2 \lambda}} \int_{0}^{\infty} e^{-\sqrt{2 \lambda}(y+x)} g(y) d y
\end{aligned}
$$

where $H(z)=(z-1) /(z+1)$. Observe that for $f \equiv 1$ we have

$$
\begin{aligned}
\left\|\lambda\left(\lambda-A_{\varepsilon}\right)^{-1} f\right\|_{L} & =\sup _{x \in \mathbb{R}^{+}}\left|\frac{1}{2}-\frac{1}{2}\left(1-e^{-\sqrt{2 \lambda} x}\right)+\frac{1}{2} H(\varepsilon \sqrt{2 \lambda}) e^{-\sqrt{2 \lambda} x}\right| \\
& =\frac{1}{2}|1+H(\varepsilon \sqrt{2 \lambda})| \underset{\lambda \rightarrow 0}{\longrightarrow} 0 .
\end{aligned}
$$

Furthermore, if $f \in \mathcal{L}^{1}\left(\mathbb{R}^{+}\right) \cap L$, then

$$
\left\|\lambda\left(\lambda-A_{\varepsilon}\right)^{-1} f\right\|_{L} \leq \sqrt{\frac{\lambda}{2}}\left[\|f\|_{\mathcal{L}^{1}\left(\mathbb{R}^{+}\right)}+|H(\varepsilon \sqrt{2 \lambda})| \cdot\|f\|_{\mathcal{L}^{1}\left(\mathbb{R}^{+}\right)}\right] \underset{\lambda \rightarrow 0}{\longrightarrow} 0,
$$

which means that $\lim _{\lambda \rightarrow 0}\left(\lambda-A_{\varepsilon}\right)^{-1} f \equiv 0$ on a dense subset of $L$ and therefore on the whole of $L$. Thus, by Proposition 10, $\lim _{t \rightarrow \infty} S_{\varepsilon}(t) f=0$, in agreement with the probabilistic interpretation of the semigroups $\left\{S_{\varepsilon}(t): t \geq 0\right\}$, $\varepsilon>0$ ([33], p. 45). An analogous argument shows that the semigroup $\{S(t): t \geq 0\}$ related to the reflecting Brownian motion ([33], p. 40), i.e. the semigroup generated by the operator $A$ with $\mathcal{D}(A)=\{f \in L$ : $\left.f^{\prime \prime} \in L, f^{\prime}(0)=0\right\}$, satisfies $\lim _{t \rightarrow \infty} S(t) f=P f$ where $P$ is the projection onto the one-dimensional subspace generated by $f_{0} \equiv 1$, given by $\operatorname{Pf}(\cdot)=\lim _{t \rightarrow \infty} f(t)$.

Let us also mention here the paper [39] where the asymptotic stability of semigroups acting in spaces of continuous functions was considered.

Let us remark that Proposition 10 cannot be extended to the case when $\{T(t): t \geq 0\}$ is differentiable without making additional assumptions on the spectrum of its generator. This is shown by the simple example of the semigroup $T(t) z=e^{i t} z$ acting in $\mathbb{C}$.

Furthermore, our Proposition 10 is worth comparing with the well-known result appearing e.g. in [48], p. 118, stating that if $A$ is the generator of a holomorphic semigroup $\{T(t): t \geq 0\}$ and $\sup \{\operatorname{Re} \lambda: \lambda \in \sigma(A)\}<0$, then $\{T(t): t \geq 0\}$ decays exponentially (see [52] for an extension of this result). Our theorem states in particular that if for a holomorphic semigroup 
condition (iii) of Proposition 8 is satisfied and additionally $\operatorname{Ker} A=\{0\}$, then $\lim _{t \rightarrow \infty} T(t) f=0$, but in general the decay is not exponential.

A few remarks on the relation to the generalization of the theorem of Sklyar and Shirman [51], obtained independently by Lyubich and Phóng [44] and Arendt and Batty [5] (see also [6-7] for recent results in this field) are also in order. Sklyar and Shirman have proved that if the spectrum of a bounded and dissipative operator $A$ has at most countable intersection with the imaginary axis and $A^{*}$ has no imaginary eigenvalues, then $\lim _{t \rightarrow \infty}\left\|e^{t A} f\right\|=0$, and in $[5,44]$ this result has been extended to the case when $A$, instead of being bounded and dissipative, is the infinitesimal generator of a semigroup of equibounded operators. Of course, our assumptions imply that the spectrum of the generator $A$ intersects the imaginary axis in at most one point, but the rest of the Sklyar-Shirman-Lyubich-PhóngArendt-Batty assumptions are not satisfied, as seen e.g. from the example of the semigroup related to the reflecting Brownian motion, mentioned in Example 3 above.

Furthermore, let us recall that, according to [20], Corollary 5.2, p. 126, if $L$ is a reflexive Banach space, then condition (iii) in Proposition 8 is automatically satisfied. This proves the following corollary ([25], p. 61, Exercise 17, cf. [45], p. 345).

Proposition 9. If $\{T(t): t \geq 0\}$ is a holomorphic semigroup of equibounded operators acting in a reflexive Banach space $L$, then for all $f \in L$ the limit $\lim _{t \rightarrow \infty} T(t) f$ exists.

A number of examples of analytic semigroups acting in reflexive Banach space can be found in the recent paper [24], and many others can be constructed by using semigroups generated by bilinear forms, as presented e.g. in $[36]$.

\section{References}

[1] N. H. Abdelazis, A note on convergence of linear semigroups of class (1.A), Hokkaido Math. J. 18 (1989), 513-521.

[2] —, On approximation by discrete semigroups, J. Approx. Theory 73 (1993), 253-269.

[3] N. H. Abdelazis and P. R. Chernoff, Continuous and discrete semigroup approximations with applications to the Cauchy problems, J. Operator Theory 32 (1994), 331-352.

[4] W. Arendt, Vector-valued Laplace transforms and Cauchy problem, Israel J. Math. 59 (1987), 321-352.

[5] W. Arendt and C. J. K. Batty, Tauberian theorems and stability of one-parameter semigroups, Trans. Amer. Math. Soc. 306 (1988), 837-852.

[6] C. J. K. Batty, Some Tauberian theorems related to operator theory, in: Banach Center Publ. 30, Inst. Math., Polish Acad. Sci., 1994, 21-34. 
[7] C. J. K. Batty, Asymptotic behaviour of semigroups, ibid., 35-52.

[8] B. Bäumer and F. Neubrander, Laplace transform methods for evolution equations, Confer. Sem. Mat. Univ. Bari 1994, 27-60.

[9] A. Bobrowski, Degenerate convergence of semigroups, Semigroup Forum 49 (1994), 303-327.

[10] - Examples of a pointwise convergence of semigroups, Ann. Univ. Mariae CurieSkłodowska Sect. A 49 (1995), 15-33.

[11] - Integrated semigroups and the Trotter-Kato theorem, Bull. Polish Acad. Sci. Math. 41 (1994), 297-304.

[12] - On the generation of non-continuous semigroups, Semigroup Forum 54 (1997), 237-252.

[13] - On the Yosida approximation and the Widder-Arendt representation theorem, Studia Math. 124 (1997), 281-290.

[14] - On approximation of (1.A) semigroups by discrete semigroups, Bull. Polish Acad. Sci. Math. 46 (1998), 141-154.

[15] S. Busenberg and B. Wu, Convergence theorems for integrated semigroups, Differential Integral Equations 5 (1992), 509-520.

[16] J. T. Cannon, Convergence criteria for a sequence of semi-groups, Appl. Anal. 5 (1975), 23-31.

[17] P. J. Cohen, Factorization in group algebras, Duke Math. J. 26 (1959), 199-205.

[18] P. C. Curtis and A. Figà-Talamanca, Factorization theorems for Banach algebras, in: Function Algebras, F. T. Birtel (ed.), Scott and Foresman, Chicago, 1966.

[19] G. Da Prato and E. Sinestrari, Differential operators with non-dense domain, Ann. Scuola Norm. Sup. Pisa 14 (1987), 285-344.

[20] E. B. Davies, One-Parameter Semigroups, Academic Press, London, 1980.

[21] B. D. Doytchinov, W. J. Hrusa and S. J. Watson, On perturbation of differentiable semigroups, Semigroup Forum 54 (1997), 100-111.

[22] N. Dunford, Spectral theory, I. Convergence to projections, Trans. Amer. Math. Soc. 54 (1943), 185-217.

[23] S. N. Ethier and T. G. Kurtz, Markov Processes. Characterization and Convergence, Wiley Ser. Probab. Math. Statist., Wiley, New York, 1986.

[24] A. Favini, J. A. Goldstein and S. Romanelli, Analytic semigroups on $L_{w}^{p}$ and on $L^{p}(0,1)$ generated by classes of second order differential operators, preprint, 1997.

[25] J. A. Goldstein, Semigroups of Linear Operators and Applications, Oxford Math. Monographs, 1985.

[26] J. A. Goldstein, C. Radin and R. E. Showalter, Convergence rates of ergodic limits for semigroups and cosine operator functions, Semigroup Forum 16 (1978), 89-95.

[27] E. Görlich and D. Pontzen, Approximation of operator semigroups of Oharu's class $C_{(k)}$, Tôhoku Math. J. (2) 34 (1982), 539-552.

[28] S. L. Gulik, T. S. Liu and A. C. M. van Rooij, Group algebra modules II, Canad. J. Math. 19 (1967), 151-173.

[29] B. Hennig and F. Neubrander, On representations, inversions and approximations of Laplace transform in Banach spaces, Appl. Anal. 49 (1993), 151-170.

[30] E. Hewitt, The ranges of certain convolution operators, Math. Scand. 15 (1964) $147-155$.

[31] E. Hille, On the differentiability of semigroups of operators, Acta Sci. Math. (Szeged) 12 (1950), 19-24. 
[32] E. Hille and R. S. Phillips, Functional Analysis and Semi-Groups, rev. ed., Amer. Math. Soc. Colloq. Publ. 31, Providence, R.I., 1957.

[33] K. Ito and H. P. Mc Kean, Jr., Diffusion Processes and Their Sample Paths, Springer, Berlin, 1965.

[34] T. Kato, Perturbation Theory for Linear Operators, Springer, New York, 1966.

[35] J. Kisyński, A proof of the Trotter-Kato theorem on approximation of semigroups, Colloq. Math. 18 (1967), 181-184.

[36] - Semigroups of operators and some of their applications to partial differential equations, in: Control Theory and Topics in Functional Analysis, Vol. 3, IAEA, Vienna, 1978, 305-405.

[37] T. G. Kurtz, Extensions of Trotter's operator semigroup approximation theorems, J. Funct. Anal. 3 (1969), 354-375.

[38] - A general theorem on the convergence of operator semigroups, Trans. Amer. Math. Soc. 148 (1970), 23-32.

[39] A. Lasota and R. Rudnicki, Asymptotic behaviour of semigroups of positive operators on $C(X)$, Bull. Polish Acad. Sci. Math. 36 (1988), 151-159.

[40] C. Lizama, On the convergence and approximation of integrated semigroups, J. Math. Anal. Appl. 181, (1994), 89-103.

[41] G. Lumer, Solutions généralisées et semi-groupes intégrés, C. R. Acad. Sci. Paris Sér. I 310 (1990), 557-582.

[42] - , A (very) direct approach to locally Lipschitz continuous integrated semigroups and some related new results oriented towards applications, via generalized solutions, in: LSU Seminar Notes in Functional Analysis and PDEs, 1990-1991, Louisiana State Univ., Baton Rouge, 1991, 88-107.

[43] —, Evolution equations: Solutions for irregular evolution problems via generalized solutions and generalized initial values. Applications to periodic shocks models, Ann. Univ. Sarav. Ser. Math. 5 (1994), no. 1, 1-102.

[44] Yu. I. Lyubich and Vũ Quôc Phóng, Asymptotic stability of linear differential equations on Banach spaces, Studia Math. 88 (1988), 37-42.

[45] R. Nagel, One-Parameter Semigroups of Positive Operators, Lecture Notes in Math. 1184, Springer, Berlin, 1986.

[46] J. Neveu, Théorie des semi-groupes de Markov, Univ. Calif. Publ. Statist. 2 (1958), 319-394.

[47] A. Pazy, On the differentiability and compactness of semigroups of linear operators, J. Math. Mech. 17 (1960), 1131-1141.

[48] - Semigroups of Linear Operators and Applications to Partial Differential Equations, Appl. Math. Sci. 44, Springer, New York, 1983.

[49] R. S. Phillips, An inversion formula for the Laplace transform and semigroups of linear operators, Ann. of Math. 59 (1954), 325-356.

[50] M. Renardy, On the stability of differentiability of semigroups, Semigroup Forum 51 (1995), 343-346.

[51] G. M. Sklyar and V. Ya. Shirman, On the asymptotic stability of a linear differential equation in a Banach space, Teor. Funktsiŭ Funktsional. Anal. i Prilozhen. 37 (1982), 127-132 (in Russian).

[52] M. Slemrod, Asymptotic behaviour of $C_{0}$ semi-groups as determined by the spectrum of the generator, Indiana Univ. Math. J. 25 (1976), 783-792.

[53] T. Takahashi and S. Oharu, Approximation of operator semigroups in a Banach space, Tôhoku Math. J. 24 (1972), 505-528. 
[54] K. Yosida, On the differentiability of semigroups of linear operators, Proc. Japan Acad. 34 (1958), 337-340.

[55] -, Functional Analysis, Springer, Berlin, 1968.

Chair of Mathematics

Department of Electrical Engineering

Lublin Technical University

Nadbystrzycka 38A

20-618 Lublin, Poland

E-mail: adambob@antenor.pol.lublin.pl

Reçu par la Rédaction le 28.4.1997 